\title{
Traduire
}

Revue française de la traduction

\section{Le DiCoInfo - Méthodologie pour une nouvelle génération de dictionnaires spécialisés}

\section{Marie-Claude L'Homme}

\section{(2) OpenEdition}

\section{Journals}

Édition électronique

URL : http://journals.openedition.org/traduire/966

DOI : $10.4000 /$ traduire.966

ISSN : 2272-9992

\section{Éditeur}

Société française des traducteurs

\section{Édition imprimée}

Date de publication : 1 juin 2008

Pagination : 78-103

ISSN : 0395-773X

\section{Référence électronique}

Marie-Claude L'Homme, «Le DiCoInfo - Méthodologie pour une nouvelle génération de dictionnaires

spécialisés », Traduire [En ligne], 217 | 2008, mis en ligne le 01 juin 2008, consulté le 30 avril 2019

URL : http://journals.openedition.org/traduire/966 ; DOI : 10.4000/traduire.966 


\title{
Le DiCoInfo - Méthodologie pour une nouvelle génération de dictionnaires spécialisés
}

\author{
Marie-Claude L'Homme \\ Observatoire de linguistique Sens-Texte (OLST) \\ Université de Montréal
}

\section{Introduction}

Le travail décrit dans les pages qui suivent découle d'une série de questions qui surgissent lors du traitement des termes apparaissant dans les textes spécialisés. Ces questions peuvent être liées à la compréhension des concepts que dénotent les termes, à la recherche de leur équivalent dans une langue cible ou, encore, à la recherche de données linguistiques permettant d'insérer correctement les termes dans des textes.

Les dictionnaires spécialisés (et on peut en dire autant des banques de terminologie) sont généralement conçus pour fournir des réponses aux questions liées à la compréhension de concepts. En plus de contenir des définitions explicatives, ils fournissent des solutions d'équivalence toutes faites pour des termes de nature nominale (des noms simples et des syntagmes nominaux, ces derniers étant parfois très longs). Cependant, les renseignements de nature linguistique - alors qu'ils représentent une part importante des articles se trouvant dans les dictionnaires généraux - font souvent défaut aux dictionnaires spécialisés(1). Nous

(1) On trouve, dans la littérature terminologique, de nombreux plaidoyers en faveur d'une meilleure prise en compte des aspects linguistiques des termes, mais peu de descriptions réelles ont été conçues à ce jour. Quelques exceptions méritent toutefois d'être signalées (pour le français) : en plus du DiCoInfo qui fait l'objet du présent article, le Dictionnaire d'apprentissage du français des affaires (Binon et al. 2000) fournit de longues descriptions tenant compte du comportement linguistique des termes. 
donnons, ci-dessous, quelques illustrations des questions soulevées par cette absence(2) :

- Parties du discours autres que le nom : des verbes comme configurer, programmer et compiler sont généralement répertoriés dans les dictionnaires décrivant les termes des domaines de l'informatique ; toutefois, l'utilisateur éprouvera des difficultés à retrouver des verbes comme annuler, lancer ou tourner. Il en va de même pour des termes appartenant à la partie du discours de l'adjectif. Virtuel et binaire risquent d'apparaître dans la nomenclature d'un dictionnaire d'informatique; mais l'accès à robuste ou à puissant peut soulever des problèmes. En ce qui concerne les adjectifs, ceux-ci sont souvent répertoriés en tant que composante d'un syntagme nominal. Par exemple, virtuel pourra apparaître dans réalité virtuelle, banque virtuelle, visite virtuelle, etc. plutôt que dans un article à part entière.

- Distinctions sémantiques fines : les dictionnaires d'informatique distinguent rarement les sens d'activité et de résultat de nombreuses nominalisations de verbes, comme configuration (1. Activité qui consiste à préparer une composante matérielle ou logicielle pour qu'elle fonctionne en conformité avec une autre composante informatique ; 2 . Ensemble des propriétés rattachées à l'état d'une composante matérielle ou logicielle qui a fait l'objet d'une préparation pour qu'elle fonctionne en conformité avec une autre composante informatique). De même, même s'ils jugeront opportun de recenser l'adjectif viral, les dictionnaires d'informatique ne distingueront pas forcément les acceptions suivantes : 1. Qui comporte un virus (ex. code, message viral); 2. Par un virus (ex. attaque, menace virale).

- Comportement syntaxique des termes : peu de dictionnaires décrivent les structures syntaxiques dans lesquelles s'utilisent les termes. Or, ces renseignements sont extrêmement importants, notamment pour les verbes. Ainsi, en informatique, un utilisateur éprouvera souvent des difficultés à accéder à des renseignements lui permettant

(2) Le lecteur trouvera d'autres détails sur cette question dans L'Homme (1998, 2004). 
de connaître le régime syntaxique du verbe programmer ${ }_{1}$ ("Écrire un programme ") qui s'emploie tour à tour dans des phrases où apparaît le complément d'objet direct et d'autres où ce dernier est omis (ex. Patrick et Vincent programment en Perl; pour programmer ce genre d'application...). De même, on distinguera rarement les régimes différents de verbes rattachés à des sous-sens différents, même si ces alternances sont régulières dans un domaine donné : par exemple, démarrer ${ }_{1 a}$ (ex. l'ordinateur démarre) et démarrer $_{1 b}$ (ex. l'utilisateur démarre l'ordinateur) ; ou imprimer $1 a$ (ex. l'imprimante imprime le fichier) et imprimer ${ }_{1 b}$ (ex. l'utilisateur imprime le fichier au moyen de cette imprimante).

- Combinatoire des termes(3) : peu de dictionnaires spécialisés décrivent les combinaisons lexicales typiques dans lesquelles se retrouvent les termes. Pourtant, il s'agit là de renseignements recherchés par une catégorie d'utilisateurs importante, à savoir les traducteurs. Ainsi, l'utilisateur d'un dictionnaire d'informatique pourra difficilement accéder à l'information lui permettant de connaître les collocatifs verbaux utilisés de préférence avec données, fichier, Internet ou logiciel pour exprimer l'idée d'" utiliser ", à savoir manipuler des données, modifier ou éditer un fichier, naviguer dans Internet et utiliser un logiciel. De même, rien ne lui permettra d'accéder aux verbes qui expriment cette fois-ci l'idée de " commencer à utiliser ... ", à savoir lancer et se connecter ou accéder, combinés à logiciel et à Internet respectivement.

Même si nous avons illustré les lacunes linguistiques des dictionnaires spécialisés existants au moyen d'exemples français, les remarques faites ci-dessous s'appliquent aussi bien à d'autres langues. Peu de dictionnaires spécialisés, quelle que soit la langue ou la paire de langues envisagée ( $s$ 'il s'agit de dictionnaires bilingues), se focalisent sur le type de renseignements que nous avons énumérés ci-dessus.

(3) Ici encore, nous pouvons signaler quelques exceptions pour le français : Lexique de cooccurrents (Cohen 1986) ; Internet. Répertoire bilingue de combinaisons lexicales spécialisées français-anglais (Meynard 2000). 
Afin d'apporter quelques éléments de solution aux problèmes évoqués dans cette section, nous nous sommes attaqués à l'élaboration d'un dictionnaire spécialisé portant sur l'informatique et sur l'Internet, appelé DiCoInfo Dictionnaire fondamental de l'informatique et de l'Internet. Suivant Sager (1990), nous partons du principe que la terminologie est une discipline regroupant une série d'activités reliées à la collecte, à la description, au traitement et à la représentation de termes(4) et que la théorie terminologique sert à résoudre les problèmes soulevés par cette pratique.

Nous nous attacherons, dans le présent article, à décrire la méthodologie qui préside à la rédaction des articles. Mais, auparavant, nous donnons un aperçu du contenu du dictionnaire. Dans cette présentation, nous nous focalisons sur les aspects généraux de la description en écartant volontairement les détails formels et techniques(5).

\section{Un aperçu du DiCoInfo}

Le DiCoInfo a d'abord été développé en français et une version est accessible en ligne (www.olst.umontreal.ca/dicoinfo). Des versions anglaise et coréenne(6) sont en cours de construction et l'accès à certains articles est prévu pour l'été 2008. Enfin, une version espagnole sera mise au point avec la collaboration de l'équipe TecnoLeTTra de l'Université Jaume I, de Castellón (Espagne). Dans cette section, nous ferons davantage référence au français, puisque cette version est la plus avancée. Toutefois, dans la section consacrée à la méthodologie, nous donnerons des exemples de termes décrits ou à décrire dans les autres langues.

(4) "Terminology is the study of and the field of activity concerned with the collection, description, processing and presentation of terms, i.e. lexical items belonging to specialised areas of usage in one or more languages" (Sager $1990: 2$ ).

(5) Le lecteur qui souhaite en savoir davantage peut se reporter à L'Homme (2008).

(6) La version coréenne est développée par Hee Sook Bae et ses travaux sont décrits dans Bae et L'Homme (2008). 
Le dictionnaire répertorie des termes fondamentaux dans le domaine de l'informatique et de l'Internet. Les termes retenus appartiennent aux parties du discours du nom (ex. données, ordinateur ; anglais, printer, virus ; espagnol, servidor, archivo), du verbe (ex. afficher, naviguer ; anglais, infect, activate ; espagnol, seleccionar, pulsar), de l'adjectif (ex. robuste, virtuel ; anglais, compatible, free ; espagnol, digital, gráfico) et de l'adverbe (ex. numériquement, dynamiquement). Le dictionnaire répertorie également des locutions (ex. système d'exploitation, par défaut; anglais, word processor; espagnol, base de datos).

Chaque article du dictionnaire correspond à un sens spécifique. Le DiCoInfo ne retient que les acceptions jugées opportunes pour les domaines de l'informatique et de l'Internet, même si d'autres acceptions peuvent se retrouver dans les corpus analysés (voir la section consacrée à la méthodologie, notamment la section 3.3 portant sur les distinctions sémantiques). Ainsi, deux articles sont consacrés à courriel (courriel ${ }_{1}: j$ 'ai transmis un courriel à mon patron pour l'informer de ma démission; courriel $_{2}$ : le courriel que vous recevez chaque jour peut être redirigé vers cette boîte). De même, deux articles distincts contiennent l'information reliée au verbe valider (valider ${ }_{1}$ : vous pouvez valider en appuyant sur ce bouton; valider $_{2}$ : valider le code $\left.H T M L\right)$.

Les articles du DiCoInfo se déclinent en une dizaine de rubriques. Comme le montre la figure 1, les rubriques Entrée, Information grammaticale, Statut (correspondant à l'état d'avancement de la rédaction de l'article), Structure actancielle, Définition (pour les termes de statut 0) et Synonymes apparaissent systématiquement dans l'affichage par défaut (de même, de l'information administrative, à savoir le rédacteur ainsi que la date de la dernière mise à jour, est donnée).

D'autres rubriques sont affichées à la demande de l'utilisateur. C'est le cas, notamment, des rubriques Contextes et Liens lexicaux. Certains articles comportent également une rubrique intitulée Informations complémentaires : cette dernière mène l'utilisateur vers des sites contenant de l'information encyclopédique. La rubrique Contextes fournit quelques exemples d'utilisation des termes dans des phrases 
extraites du corpus utilisé pour élaborer le dictionnaire. La rubrique Liens lexicaux, quant à elle, donne une liste d'unités lexicales partageant avec le terme apparaissant en entrée un lien de nature sémantique. De plus, elle fournit une explication sur la nature de ce lien. Lorsque la description du terme relié est donnée dans le dictionnaire, un hyperlien dirige l'utilisateur vers elle. Nous avons reproduit, à la figure 2, la liste de liens lexicaux pour le terme barre d'espacement ${ }_{1}$.

barre d'espacement 1 , n. f.

Statut : 0

Structure actancielle : la barre d'espacement : utilisée par AGENT \{utilisateur 1\} pour intervenir sur PATIENT \{espace 2\}(7)

Réalisations linguistiques des actants

Définition : Touche de forme allongée placée au bas du clavier qu'un UTILISATEUR utilise afin d'insérer des ESPACES.

Synonyme(s) : barre espace, barre d'espace

Contextes

Liens lexicaux

Figure 1. Article barre d'espacement

(7) Le formalisme utilisé dans la structure actancielle est expliqué à la section 3.2.2. 
barre d'espacement ${ }_{1}, \mathrm{n} . \mathrm{f}_{\text {. }}$

Statut : 0

$\underline{\text { Liens lexicaux }}$

\begin{tabular}{|c|c|}
\hline Explication - terme typique & Lexie reliée \\
\hline$\sim(8)$ & touche $_{1}$ \\
\hline$\sim$ & pavé $_{1}$ \\
\hline$\sim$ & flèche $_{1}$ \\
\hline L'utilisateur utilise la b. & appuyer $_{1}$ sur la \\
\hline L'utilisateur utilise la b. & enfoncer ${ }_{1} l a$ \\
\hline$\rightarrow \operatorname{NOM}(9)$ & enfoncement ${ }_{1}$ de la - \\
\hline $\begin{array}{l}\text { L'utilisateur utilise la b. pour intervenir } \\
\text { sur un espace }\end{array}$ & insérer $_{2} \ldots$ avec la \\
\hline$\rightarrow \mathrm{NOM}$ & insertion $_{2}$ de ... avec la \\
\hline L'utilisateur cesse d'utiliser la b. & relâcher $_{1}$ la \\
\hline$\rightarrow$ NOM & relâchement $t_{1}$ de la - \\
\hline Lieu où on trouve une b. & clavier $_{1}$ \\
\hline Lieu où on trouve une $b$. & mini-clavier $_{1}$ \\
\hline
\end{tabular}

Figure 2. Liens lexicaux de barre d'espacement

La version en ligne du DiCoInfo permet l'accès à un peu plus de 1000 articles. En outre, plus de 20000 liens lexicaux sont décrits pour ces termes, ce qui représente en moyenne 20 liens lexicaux par terme. Un module de recherche permet à l'utilisateur d'accéder aux termes apparaissant en entrée, mais également aux multiples liens lexicaux présents dans chacun des articles. Enfin, une version statique est également proposée.

(8) Le symbole est utilisé pour rendre compte de l'ensemble des sens voisins, à savoir la quasi-synonymie, l'hyperonymie, la co-hyponymie, etc.

(9) => NOM est utilisé pour décrire le lien existant entre le terme décrit et une nominalisation de verbe. Puisque le verbe lui-même apparaît juste au-dessus, nous ne reprenons pas toute l'explication. 


\section{Méthodologie(10)}

La méthodologie mise au point pour élaborer les articles comprend un certain nombre de tâches regroupées en deux catégories distinctes. La première, la collecte de données et l'établissement de la nomenclature, comprend :

1) l'élaboration d'un corpus ;

2) la sélection de termes ;

3) l'établissement de distinctions sémantiques.

La section étape comprend des tâches reliées plus spécifiquement à la rédaction des articles, notamment :

1) la définition de la structure actancielle ;

2) l'établissement de la liste de liens lexicaux.

Les tâches rattachées à ces étapes sont réalisées de façon indépendante dans chacune des langues. Léquivalence formelle entre les sens sera réalisée une fois la rédaction des articles suffisamment avancée dans les langues autres que le français.

\subsection{Collecte de données et établissement de la nomenclature}

\subsubsection{Corpus}

La sélection ainsi que l'analyse des termes repose en premier lieu sur les observations faites par les terminologues dans un corpus spécialisé. Le tableau 1 donne les détails des corpus utilisés jusqu’à présent pour chacune des langues (certains corpus, notamment le corpus espagnol,

(10) Même si nous n'insistons pas ici sur les aspects théoriques du DiCoInfo, il importe de souligner que sa confection s'appuie principalement sur les modèles et la méthodologie mis au point dans le cadre de la Lexicologie explicative et combinatoire (LEC) (Mel'Čuk et al., 1995). Il emprunte également des éléments théoriques et méthodologiques à Cruse (1986) ainsi qu'à la sémantique des cadres ou Frame semantics (Fillmore, 1982 ; Fillmore et al., 2003) tels qu'ils peuvent s'observer dans la ressource FrameNet (2008). 
doivent être enrichis). À noter que tous ces corpus, à l'exception du corpus coréen qui était disponible, ont été élaborés pour le DiCoInfo.

Les corpus décrits dans le tableau 1 constituent un point de départ pour les terminologues chargés de sélectionner et d'analyser les termes. Ils les consultent au moyen de fonctions implémentées dans des concordanciers (recherche de formes tronquées, de combinaisons de termes, etc.). Toutefois, aucun corpus ne renferme tous les éléments nécessaires à l'appréhension des sens des termes et à l'examen de l'ensemble des liens qu'ils partagent avec d'autres termes du domaine. Le terminologue fait également appel à l'expertise de spécialistes ainsi qu'à son intuition, intuition qu'il affûte au fur et à mesure de l'avancement des travaux. Enfin, afin de retrouver l'ensemble des liens lexicaux rattachés à un terme, une recherche dans des corpus nettement plus volumineux se révèle souvent nécessaire.

Tableau 1 : Corpus utilisés en anglais, coréen, espagnol et français

\begin{tabular}{|l|l|l|l|l|}
\hline Langue & Occurrences & Spécialisation & $\begin{array}{c}\text { Variantes } \\
\text { régionales }\end{array}$ & Provenance \\
\hline Anglais & Env. 1 million & $\begin{array}{l}\text { Didactique, } \\
\text { vulgarisation }\end{array}$ & $\begin{array}{l}\text { Textes états-uniens } \\
\text { principalement }\end{array}$ & Internet \\
\hline Coréen & $\begin{array}{l}4230000 \\
\text { eojeols }(11)\end{array}$ & Spécialisé & $\begin{array}{l}\text { Information } \\
\text { non disponible }\end{array}$ & $\begin{array}{l}\text { Corpus élaboré } \\
\text { par le centre de } \\
\text { recherche KAIST } \\
\text { (Séoul) }\end{array}$ \\
\hline Espagnol & Env. 500 000 & $\begin{array}{l}\text { Didactique, } \\
\text { vulgarisation }\end{array}$ & $\begin{array}{l}\text { Textes espagnols ; } \\
\text { différentes variétés } \\
\text { latino-américaines }\end{array}$ & Internet \\
\hline Français & Env. 2 millions & $\begin{array}{l}\text { Didactique, } \\
\text { vulgarisation }\end{array}$ & $\begin{array}{l}\text { Textes français } \\
\text { principalement ; } \\
\text { quelques textes } \\
\text { québécois }\end{array}$ & $\begin{array}{l}\text { Quelques textes } \\
\text { numérisés ; } \\
\text { Internet }\end{array}$ \\
\hline
\end{tabular}

(11) En coréen, l'eojeol correspond à une unité séparée des autres par des blancs typographiques : il s'agit, dans la plupart des cas, d'un mot lexical combiné à un mot grammatical. 


\subsubsection{Sélection des termes}

La sélection des termes repose sur l'utilisation d'un extracteur de termes combinée à une analyse, par les terminologues, des résultats qu'il propose. L'extracteur terminologique utilisé, appelé TermoStat (Drouin 2003), génère une liste de candidats à partir d'un calcul permettant d'évaluer la spécificité des unités en opposant leurs occurrences dans un corpus spécialisé à celles observées dans un autre corpus, appelé corpus de référence.

Pour les fins du DiCoInfo, le calcul est appliqué à toutes les formes simples (à noter que TermoStat peut également être utilisé pour retrouver des syntagmes nominaux).

Le tableau 2 montre les 10 premiers termes spécifiques obtenus pour l'anglais, le coréen, l'espagnol et le français en utilisant cette technique. Même si ces premiers candidats ne sont donnés ici qu'à titre indicatif (les résultats ont été produits à partir de corpus très différents tant sur le plan de la taille que sur celui du contenu), il est intéressant de noter que certains termes (ex. fichier et ses équivalents; logiciel et des termes de sens voisin) se retrouvent en tête de liste dans les quatre cas.

Tableau 2 : Premiers candidats spécifiques valides anglais, espagnols et français

\begin{tabular}{|l|l|l|l|}
\hline Anglais & Coréen & Espagnol & Français \\
\hline Use & 사용자 'utilisateur' & servidor & Fichier \\
\hline File & 시스템 'système' & usuario & commande \\
\hline program & 메시지 'message' & cliente & Internet \\
\hline computer & 프로세스 'processus' & procesador & Serveur \\
\hline Server & 파일 'fichier' & archivo & Utiliser \\
\hline Web & 검색 'recherche' & computadora & utilisateur \\
\hline Install & 경로 'répertoire' & pantalla & Logiciel \\
\hline System & 호스트 'hôte' & soporte & Option \\
\hline User & 웹 'Web' & herramienta & ordinateur \\
\hline Windows & 소프트웨어 'logiciel' & disco & Système \\
\hline
\end{tabular}


Les listes de candidats sont ensuite étudiées par les terminologues qui sélectionnent les noms, verbes, adjectifs ou adverbes valables, c'est-àdire qui feront l'objet d'un ou de quelques articles dans le dictionnaire. Ils s'aident dans cette tâche des quatre critères suivants :

- Dénotation d'une entité liée au domaine : ex. fichier, file, archivo. Ce premier critère, de nature extra-linguistique, est celui qui est le plus souvent utilisé par les terminologues adoptant des méthodes d'obédience conceptuelle. Le lien entre une unité lexicale et une réalité du monde réel est plus facilement établi entre un terme de nature nominale et des entités (à des objets concrets ou à des animés, par exemple). L'identification de termes qui dénotent d'autres réalités (par exemple, des activités ou des propriétés) doit reposer sur la prise en compte de leur nature linguistique. Les terminologues du DiCoInfo ont donc recours aux trois critères suivants :

- Présence d'actants de nature spécialisée : les termes dénotant des activités ou des propriétés sont par essence de nature prédicative (c'est-à-dire que leur sens fait appel à des actants). Ce deuxième critère permet de saisir le caractère terminologique d'une unité lexicale en tenant compte non seulement de l'unité de nature prédicative, mais de ses actants. Il s'agit plus concrètement de voir si l'unité lexicale fait appel à des actants de nature spécialisée (en fonction du critère 1). Si oui, l'unité prédicative est probablement un terme ; dans le cas contraire, le statut terminologique de l'unité doit être remis en cause. Par exemple, le terme espagnol guardar s'emploie avec des termes d'informatique, à savoir archivo, datos, documento, d'une part, et disco duro, disco, servidor, d'autre part. La nature des actants dans ce cas permet d'admettre guardar comme terme spécialisé.

- Lien morphologique accompagné d'un lien sémantique : ce critère permet d'admettre des unités lexicales partageant un lien morphologique (accompagné d'un lien de sens) avec une unité déjà admise comme terme (en fonction des critères 1 et 2). Par exemple, les termes anglais to compile et to recompile, to precompile, compilable seront retenus si compiler a été sélectionné. 
- Autre lien paradigmatique : enfin, l'existence de liens paradigmatiques (autres que ceux également accompagnés d'un lien morphologique vérifié au moyen du critère précédent) permet d'admettre de nouvelles unités. Par exemple, supprimer et effacer seront retenus puisque leur antonyme, à savoir créer, est sélectionné.

Lors de la sélection des termes, les terminologues ont souvent davantage de facilité à retenir des formes n'existant que dans le domaine faisant l'objet de l'analyse et les termes dont le (ou les) sens se distingue nettement du sens que l'unité peut avoir dans d'autres contextes. Dans ces premiers cas de figure, les critères énumérés ci-dessus seront rarement mobilisés. Par exemple, les verbes configurer ou déboguer ou l'adjectif cliquable feront invariablement l'unanimité. Toutefois, les adjectifs robuste et puissant ainsi que les verbes installer, valider ou créer soulèveront davantage de problèmes. Dans la perspective du DiCoInfo, les terminologues retiennent des unités lexicales si elles font partie du lexique du domaine décrit et non uniquement si leur sens ou leur emploi se distingue nettement de sens ou d'emplois qu'elles peuvent avoir dans d'autres contextes(12). Dans ces cas, les critères donnés dans cette section seront fort utiles pour permettre aux terminologues de voir si les unités en question font réellement partie du lexique d'un domaine.

Enfin, d'autres indices peuvent être utilisés afin d'évaluer le statut d'une unité lexicale dans un domaine. Ces indices sont la fréquence et la répartition. L'emploi fréquent d'une unité lexicale ainsi que sa présence dans de nombreux textes spécialisés différents révèlent souvent son importance dans le domaine.

(12) Cette perspective est tout à fait compatible avec celle de la pratique terminologique habituelle, bien que cette dernière soit généralement appliquée à des noms. Par exemple, on n'hésitera pas à inclure des unités comme écran et clavier dans un dictionnaire d'informatique, même si les sens de ceux-ci ne different pas des sens qu'ils ont dans d'autres contextes. 


\subsubsection{Distinctions sémantiques}

Une partie des unités lexicales de nature terminologique sélectionnées lors de l'étape précédente doivent faire l'objet de distinctions sémantiques. Dans certains cas, tous les sens sont rattachés au domaine de l'informatique (par exemple, server, en anglais, et serveur, en français renvoient à deux sens distincts : 1 . " un ordinateur jouant un rôle central dans un réseau "; 2 . "un logiciel qui se charge d'une série de tâches reliées à une application) "; dans d'autres, les sens d'une forme lexicale peuvent se répartir en acceptions informatiques et acceptions rattachées à d'autres domaines spécialisés ou à des emplois de nature générale (par exemple, le nom anglais application peut désigner : 1. « un logiciel utilisé pour exécuter des tâches rattachées à un contexte de traitement (souvent professionnel) "; 2 . "le contexte en question (par exemple, la comptabilité) "; mais on trouvera également, dans les corpus informatiques, la nominalisation du verbe apply qui n'est pas spécifiquement informatique. Ici, quatre nouveaux critères servent à baliser les intuitions des terminologues lors du tri des acceptions attestées dans les corpus spécialisées :

- Cooccurrence compatible ou différentielle (Mel'čuk et al., 1995) : ce critère sert à voir si les cooccurrents d'une unité lexicale posant problème peuvent se combiner dans une phrase normale. Si oui, l'unité lexicale n'a qu'un sens ; dans le cas contraire, elle est fort probablement polysémique. Par exemple, le verbe formater peut se combiner avec les noms disque et texte. Toutefois, la coordination des deux noms ne produit pas un énoncé acceptable en français ( ${ }^{*}$ formater un texte ou un disque dur).

- Substitution par un synonyme : ce deuxième critère sert à observer les possibilités de substitution d'une unité lexicale posant problème par une autre de même sens. Si la seconde unité peut remplacer la première dans tous les contextes analysés, l'unité faisant problème est monosémique ; si non, elle est probablement polysémique. Par exemple, le verbe espagnol seleccionar peut être remplacé par marcar lorsque le patient subissant cette action est un bloc de texte ; ce rem- 
placement n'est plus possible s'il s'agit d'une option (?marcar una opción). De même, le verbe français, valider s'emploie avec option et avec code. Toutefois, le synonyme accepter n'est valable que pour option (valider du code, ?accepter du code).

- Dérivation morphologique différentielle : ce critère permet au terminologue de voir si un dérivé morphologique s'applique à toutes les occurrences d'une unité faisant problème. Si oui, l'unité en question est fort probablement monosémique ; si non, le terminologue a probablement affaire à plus d'un sens. Par exemple, le verbe anglais install s'utilise avec program et avec computer (install a program; install your computer). Toutefois, le dérivé uninstall n'est valable que pour program (uninstall this program; ?uninstall your computer).

- Autres liens paradigmatiques différentiels : ce dernier critère sert à utiliser des liens paradigmatiques (autres que ceux évoqués jusqu'ici : la synonymie et la dérivation morphologique) pour valider des distinctions sémantiques. Par exemple, ce critère permet de distinguer les sens du nom français page qui peut être : 1. "la division d'un document"; 2. "la division d'une mémoire "; et, enfin, 3. "une partie d'un site web ». Chaque sens est validé en distinguant les holonymes (unités désignant le tout) de page, à savoir document, mémoire et site.

\subsection{Rédaction des articles}

La rédaction des articles par les terminologues se fait désormais(13) dans une structure de données $\mathrm{XML}(14)$. Les articles du dictionnaire se construisent peu à peu en différentes étapes, correspondant à l'état

(13) Auparavant, les articles étaient préparés dans une base de données relationnelle et gérés au moyen du système de gestion de bases de données Access.

(14) La conversion du DiCoInfo en format XML ainsi que l'élaboration des modes de présentation HTML et PDF ont été réalisées en collaboration avec Guy Lapalme, Benoît Alain et Vincent St-Amour. 
d'avancement de l'analyse. Par exemple, un premier terminologue peut travailler à la collecte de contextes et ne remplira que cette section de l'article ; un autre peut se pencher sur certains liens lexicaux, et ainsi de suite. Un champ appelé Statut permet de garder la trace de l'avancement de la rédaction.

Les sous-sections qui suivent décrivent les parties de la méthodologie consacrées plus spécifiquement à la rédaction des articles.

\subsubsection{Termes et contextes}

Rappelons que chaque article du DiCoInfo est consacré à la description d'un sens. Les sens ainsi distingués apparaîtront avec un numéro d'acception $(1,2$, etc.). Les sens voisins seront distingués au moyen de lettres ( $1 \mathrm{a}, 1 \mathrm{~b}$, etc.).

À noter également que le terminologue extrait, pour chaque sens retenu, des contextes dans lesquels le terme véhicule ce sens, comme l'illustre le tableau 3. 


\section{Tableau 3 : Contextes relevés pour illustrer les termes}

\begin{tabular}{|c|c|}
\hline \multirow[t]{2}{*}{ save 1} & $\begin{array}{l}\text { Then, you edit the file from another window and REDUCE } \\
\text { the file size to something less than the current size, and } \\
\text { save it. }\end{array}$ \\
\hline & $\begin{array}{l}\text { This means that the last settings that you set for the pro- } \\
\text { gram will be saved the next time you launch the same } \\
\text { program using Priority Master II. }\end{array}$ \\
\hline \multirow{3}{*}{$\begin{array}{l}\text { 저장하다 } 1 \\
\text { 'sauvegarder' }\end{array}$} & 그 정보는 실시간으로 통합서버에 저장이 된다. \\
\hline & $\begin{array}{l}\text { 'Les données sont sauvegardées en temps réel sur un ser- } \\
\text { veur d'intégration.' }\end{array}$ \\
\hline & $\begin{array}{l}\mathrm{PC} \text { 에 전송된 내용은 파일의 형태로 저장된다. 'Les } \\
\text { contenus transférés vers le PC sont sauvegardés en tant que } \\
\text { fichiers.' }\end{array}$ \\
\hline \multirow[t]{2}{*}{ seleccionar 1} & $\begin{array}{l}\text { Si quieres seleccionar un único archivo o carpeta sólo has } \\
\text { de hacer clic sobre él. }\end{array}$ \\
\hline & $\begin{array}{l}\text { Seleccionar la opción Nuevo del menú Archivo y en el } \\
\text { cuadro de diálogo que se presenta la opción Con objetos. }\end{array}$ \\
\hline \multirow[t]{2}{*}{ seleccionar 2} & $\begin{array}{l}\text { Seleccionamos una palabra pulsando dos veces sobre la } \\
\text { misma. }\end{array}$ \\
\hline & $\begin{array}{l}\text { Para actuar sobre un texto ya escrito, será necesario selec- } \\
\text { cionarlo previamente, al seleccionar una porción de texto } \\
\text { éste queda en vídeo inverso (negro). }\end{array}$ \\
\hline \multirow[t]{2}{*}{ viral la } & Microsoft n'en est pas à sa première attaque virale. \\
\hline & $\begin{array}{l}\text { Éviter la majorité des infections virales tout en protégeant } \\
\text { votre ordinateur. }\end{array}$ \\
\hline \multirow[t]{2}{*}{ viral $1 \mathrm{~b}$} & $\begin{array}{l}\text { Perrun est composé d'un troyen extracteur qui peut lire, } \\
\text { extraire et exécuter du code viral caché dans des fichiers } \\
\text { JPEG. }\end{array}$ \\
\hline & $\begin{array}{l}\text { Il se présente sous la forme d'un message incluant l'adresse } \\
\text { d'un fichier au nom... contenant un lien vers le fichier viral } \\
\text { à télécharger. }\end{array}$ \\
\hline
\end{tabular}




\subsubsection{Description de la structure actancielle}

Après avoir délimité les acceptions des termes retenus, le terminologue procède à la rédaction des articles. La première rubrique sur laquelle il se penchera est celle contenant la structure actancielle. Cette rubrique apparait sous l'entrée et l'information grammaticale. Il s'agit d'une rubrique extrêmement importante, puisqu'on en retrouve des traces dans d'autres rubriques du DiCoInfo (il en sera question, notamment dans la section 3.5 consacrée à la description des liens lexicaux). On retrouve également la mention des actants dans la définition.

La structure actancielle décrit les participants essentiels pour décrire le sens d'un terme. Il ne s'agit pas d'une définition à proprement parler, mais cette structure fournit déjà des éléments importants sur le sens des termes.

Pour commencer, le terminologue ébauche une structure en faisant appel aux connaissances qu'il a accumulées lors des étapes précédentes; ensuite, il la valide en recherchant les réalisations des actants dans les contextes. Par exemple, il pourra définir une structure actancielle contenant deux actants pour le terme configurer : quelqu'un quelque chose. Les contextes fourniront les éléments suivants pour le premier actant : informaticien, vous, administrateur, utilisateur; et les éléments suivants pour le second : carte, disque dur, imprimante, logiciel, micro-ordinateur, mémoire, application, etc. Le terminologue recueille les réalisations des actants et en dresse une liste qui sera accessible par la suite dans l'article du dictionnaire(15). Cette liste sert également à identifier le terme typique permettant à l'utilisateur du dictionnaire de se faire une idée du type de terme pouvant instancier un actant.

Le résultat de l'analyse effectuée par le terminologue prend la forme suivante (figure 3 ) pour le terme configurer :

(15) Il est à noter que, dans cette liste, le terminologue n'inscrit que les réalisations d'actants correspondant à des termes décrits dans d'autres articles. Ainsi, même si vous apparaît fréquemment dans les contextes comme réalisation du premier actant de configurer, il ne sera pas inscrit dans l'article. 
configurer $_{1}$, v. tr.

Statut 0

Structure actancielle : configurer : AGENT(16)\{utilisateur 1\} PATIENT \{logiciel 1 ; matériel 1$\}$

Réalisations linguistiques des actants

\begin{tabular}{|c|}
\hline Agent \\
\hline administrateur $_{1}$, constructeur ${ }_{1}$, informaticien ${ }_{1}$, utilisateur ${ }_{1}$ \\
\hline Patient \\
\hline 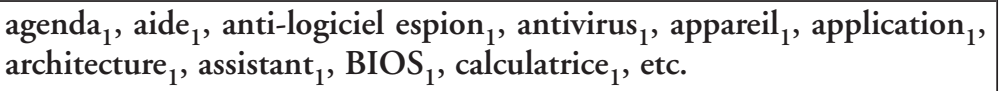 \\
\hline
\end{tabular}

Définition : un UTILISATEUR définit les paramètres de fonctionnement du MATÉRIEL ou d'un LOGICIEL pour qu'il fonctionne en conformité avec du matériel ou un logiciel spécifique.

Figure 3 : Structure actancielle et réalisations des actants du verbe configurer.

Comme nous l'avons déjà signalé plus haut, les actants sont de nouveau évoqués dans la définition, comme l'illustre la figure 3.

Pour conclure cette section, précisons que le terminologue s'assure de reproduire le parallélisme existant entre des termes de sens proche. Ce sera le cas, notamment pour les termes cliquer, clic et cliquable, dont les structures actancielles sont reproduites à la figure 4 .

(16) Le DiCoInfo note les actants au moyen de deux systèmes : le premier, en lettres majuscules (AGENT, PATIENT), rend compte des rôles sémantiques joués par les actants par rapport au terme. Nous ne nous attarderons pas sur cette notation, mais signalons qu'elle s’inspire de travaux réalisés dans le cadre de la sémantique des cadres (voir note plus haut) : l'AGENT est l'actant à l'origine de l'action ou de la création de la réalité dénotée par le terme ; le PATIENT est l'actant dénotant l'élément subissant l'action ou l'élément créé. La seconde notation, dont nous avons parlé dans cette section et qui apparaît entre accolades, présente un terme typique jouant le rôle de cet actant. Le terme typique se veut plus transparent que le rôle sémantique. 
cliquer $_{1}$, v. tr.

Statut 0

Structure actancielle : cliquer : AGENT\{utilisateur 1$\} \sim$ sur PATIENT \{icône 1 ; fichier 1 \} avec INSTRUMENT\{souris 1 \}

cliquable $_{1}$, adj.

Statut 0

Structure actancielle : cliquable : PATIENT\{icône 1 ; fichier 1$\}$ est $\operatorname{clic}_{1}$, n. m.

Statut 0

Structure actancielle : un clic : sur PATIENT\{icône 1 ; fichier 1 \} avec INSTRUMENT\{souris 1\} par AGENT\{utilisateur 1\}

Figure 4 : Structures actancielles de cliquer, cliquable et clic.

\subsubsection{Liste de liens lexicaux}

La dernière étape de la rédaction des articles du dictionnaire, la plus longue, consiste à dresser la liste de liens lexicaux. Les terminologues tentent de relever la totalité des liens paradigmatiques et syntagmatiques (c'est-à-dire les collocations) valables pour chaque terme décrit. La recherche de liens lexicaux se fait principalement de manière manuelle et consiste à combiner les stratégies suivantes :

- Identification de variantes, synonymes, de quasi-synonymes et autres sens voisins : ces liens sont souvent les premiers relevés, puisqu'ils sont utilisés lors de l'établissement de distinctions sémantiques. Le tableau 4 donne quelques exemples de ces liens pour les quatre langues décrites jusqu'à présent. Il est à noter que le lien de synonymie et les variantes graphiques n'apparaissent pas directement dans la liste de liens lexicaux dans l'article définitif, mais dans une rubrique distincte. 
Tableau 4 : Liens de synonymie et autres sens voisins

\begin{tabular}{|l|l|l|l|}
\hline Langue & Terme décrit & Lien & Terme relié \\
\hline \multirow{4}{*}{ Anglais } & Offline & Variante & off-line \\
\cline { 2 - 4 } & Return & Synonyme & carriage return \\
\cline { 2 - 4 } & Screen & Quasi-synonyme & display \\
\hline Coréen & 웹1 «Web' & Générique & 네트워크 1 « réseau » \\
\hline \multirow{3}{*}{ Espagnol } & Archivo & Synonyme & fichero \\
\cline { 2 - 4 } & Seleccionar & Quasi-synonyme & Subrayar \\
\hline \multirow{3}{*}{ Français } & Antivirus & Variante & anti-virus \\
\cline { 2 - 4 } & Fichier & Synonyme & - informatique \\
\cline { 2 - 4 } & Interrompre & Quasi-synonyme & Abandonner \\
\hline
\end{tabular}

- Identification de sens opposés : Certains termes (souvent des verbes ou des adjectifs) partagent avec d'autres termes des liens d'antonymie, de quasi-antonymie ou de contrastivité. Ces liens, tout comme les liens de synonymie et de sens voisins, sont souvent identifiés au moment d'effectuer les distinctions sémantiques.

- Recherche de liens lexicaux réalisés sous forme de termes apparentés morphologiquement : dans les corpus spécialisés, les liens morphologiques signalent souvent un lien de sens. Dans le DiCoInfo français, certaines formes apparentées indiquent un lien de sens très régulier. C'est le cas, notamment, d'un grand nombre de nominalisations véhiculant le même sens qu'un verbe (ex. sélectionner - sélection ; compiler - compilation ; configurer - configuration) ou d'adjectifs en -able signifiant " qui peut être + verbe » (ex. compiler - compilable; configurer - configurable). Pour retrouver une partie de ces liens (pour des termes qui n'ont pas été retrouvés lors de la sélection de termes), les terminologues peuvent faire appel à la fonction de recherche de formes tronquées offertes par de nombreux concordanciers (17).

(17) À noter également qu'un travail de collecte automatique de paires de termes morphologiquement apparentés a été effectué dans le cadre du DiCoInfo (Claveau et L'Homme 2005). 
- Pour les noms, recherche de liens exprimant "sorte de $\mathbf{x}$ » : une part importante de termes reliés à des noms signalent un lien " sorte de " (ex. fichier - archive, - texte, - graphique, - vide).

- Pour les noms surtout, recherche de liens de collocation : enfin, toujours en ce qui concerne les noms, la plus grande partie des liens décrits sont de nature syntagmatique. Il s'agit de combinaisons dans lesquelles entrent le terme décrit et une autre unité lexicale de nature verbale, nominale ou adjectivale. Ces liens sont énumérés et accompagnés d'un premier niveau d'explication s'inspirant de Polguère (2003). Dans le DiCoInfo, cette forme d'explication fait appel aux termes typiques apparaissant dans la structure actancielle, comme l'illustre la figure 5(18).

barre d'espacement ${ }_{1}$, n. f.

Structure actancielle : la barre d'espacement : utilisée par AGENT\{utilisateur 1\} pour intervenir sur PATIENT\{espace 2\}

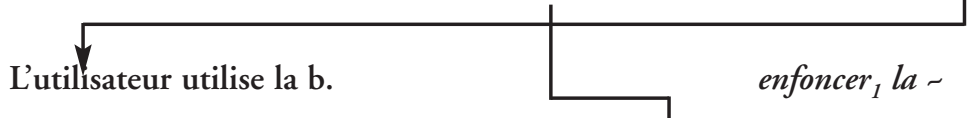

L'utilisateur utilise la b. pour intervenir sur un espace insérer ${ }_{2}$... avec la Figure 5 : Explications de liens lexicaux et structure actancielle.

Enfin, les liens lexicaux sont ordonnés dans les articles de la manière suivante :

1. Liens paradigmatiques :

a. Quasi-synonymie et autres sens voisins

b. Antonymie et autres sens opposés

c. Liens morphologiques réguliers

2. Liens "sorte de"

3. Liens de collocations verbales (et les dérivés de ces verbes) niveaux d'explication plus formels. Ceux-ci sont affichés à la demande de l'utilisateur. 
a. En ce qui concerne les liens de collocations verbales, ils sont ordonnés en fonction de l'ordre d'utilisation typique qui en est faite, comme l'illustre le tableau 5 pour le terme mot de passe.

4. Autres liens paradigmatiques (méronymie, lieu, instrument, etc.).

Tableau 5 : Quelques liens de collocations pour mot de passe mot de passe ${ }_{1}$, n. m.

Liens lexicaux

\begin{tabular}{|c|c|}
\hline Explication - terme typique & Lexie reliée \\
\hline L'utilisateur crée un $\mathrm{m}$. & choisir, créer $_{1}$, définir ${ }_{1}$ un - \\
\hline L'utilisateur cause qu'il ait un $\mathrm{m}$. & $\begin{array}{l}\text { apprendre, mémoriser, obtenir, } \\
\text { se munir d'un - }\end{array}$ \\
\hline L'utilisateur a un $\mathrm{m}$. & avoir, posséder un - \\
\hline L'utilisateur modifie un $\mathrm{m}$. & changer, modifier son - \\
\hline L'utilisateur n'a pas un $\mathrm{m}$. & oublier, perdre son - \\
\hline $\begin{array}{l}\text { L'utilisateur met un } \mathrm{m} \text {. dans } \\
\text { un endroit prévu à cette fin }\end{array}$ & entrer $_{1}$, saisir $_{1}$, taper $_{1}$ un - \\
\hline L'utilisateur utilise un $\mathrm{m}$. & utiliser $_{1}$ un \\
\hline
\end{tabular}

\section{Conclusion : enrichissements à venir du DiCoInfo}

Le DiCoInfo, on l'aura compris, est un dictionnaire en construction. Toutefois, sa rédaction est suffisamment avancée pour nous permettre d'affirmer que les articles tels qu'ils sont construits apportent des réponses aux différentes questions soulevées dans la première section de cet article : description de termes appartenant à des parties du discours diverses, prise en compte de distinctions sémantiques fines ainsi que de la combinatoire des termes. De même, la méthodologie que nous avons mise au point est, à notre avis, assez robuste pour se prêter à des extensions à de nouveaux domaines de spécialité.

Nous envisageons tout de même d'enrichir la version consultable du DiCoInfo de diverses manières. Notre conclusion consistera donc à évoquer quelques-uns des enrichissements envisagés. 
Pour l'instant, l'information sur le comportement syntaxique des termes n'est pas fournie dans une rubrique séparée : elle apparaît implicitement dans la structure actancielle. Par exemple, la structure actancielle du terme télécharger ${ }_{1}$ indique que le verbe comporte quatre actants (télécharger : AGENT\{utilisateur 1\} PATIENT\{fichier 1 ; logiciel 1\} de SOURCE\{ordinateur 1 ; réseau 1 \} à DESTINATION \{ordinateur 1\}. L'utilisateur peut inférer, à la lecture de l'article, que l'agent est normalement réalisé comme sujet, le patient, comme objet direct, la source et la destination, comme compléments reliés au verbe au moyen de prépositions spécifiques. Cependant, rien n'indique si le verbe peut admettre une construction passive, si celle-ci est la plus fréquente ou, encore, si les prépositions sont fixes ou peuvent varier. Nous comptons ajouter une nouvelle rubrique au dictionnaire qui fournira cette information. Un projet d'annotation des contextes est en cours et ses résultats seront accessibles dans un avenir rapproché.

Un autre projet en cours consiste à adapter certaines rubriques du DiCoInfo pour en produire une version papier. Comme nous l'avons souligné un peu plus haut, la structure XML peut être utilisée pour en dériver une version PDF. Nous réfléchissons actuellement à différentes manières d'organiser les rubriques afin de rendre la consultation d'un dictionnaire papier plus conviviale. Compte tenu de la richesse et de la variété des informations contenues dans les articles, il s'agit d'un exercice non trivial.

En ce qui concerne la version française du DiCoInfo, il reste environ 800 articles à mettre en ligne. Les versions anglaise et coréenne sont en cours de rédaction et nous souhaitons mettre quelques articles en ligne dans un avenir rapproché. La mise en ligne des versions dans d'autres langues posera le problème de l'établissement de liens d'équivalence entre les termes. Pour la plupart des termes, nous croyons que ces liens ne poseront pas de difficulté particulière. Dans les langues traitées jusqu'à maintenant, il existe des équivalences lexicales pour de nombreux termes appartenant à des parties du discours diverses. Toutefois, nous avons relevé quelques cas où un terme dans une langue a comme équivalent une expression non lexicale, soit une collocation ou une 
expression libre. Par exemple, le verbe français cliquer et son équivalent anglais click font tous deux l'objet d'un article dans les versions française et anglaise du DiCoInfo. Toutefois, en espagnol, la forme privilégiée pour exprimer le même sens est une collocation, à savoir hacer clic, forme qui sera décrite dans l'article consacré au nom clic. De même, le terme français cliquable se rend en coréen par 클릭할 수 있는 (littéralement "pouvant être cliqué ", donc une paraphrase construite à partir du verbe 클릭, la base du verbe cliquer). Des stratégies devront être mises au point afin de mener l'utilisateur vers des solutions de traduction valables dans chaque langue. Ces solutions différeront forcément de celles adoptées généralement dans les dictionnaires spécialisés qui consistent à fournir des équivalences toutes faites pour des séquences souvent très longues.

\section{Remerciements}

Le travail décrit dans ces pages a bénéficié du soutien financier des deux organismes subventionnaires canadiens, à savoir le Conseil de recherches en sciences humaines (CRSH) du Canada et le Fonds québécois de la recherche sur la société et la culture (FQRSC). Je tiens également à remercier des collègues et chercheurs étudiants qui ont travaillé sur les aspects du DiCoInfo décrits dans cet article : Benoît Alain, Hee Sook Bae, Louis-Philippe Dargis, Patrick Drouin, Daniel Labonia, Guy Lapalme, Chantal Lemay, Vincent St-Amour. La liste complète des contributeurs apparaît dans le site Web du DiCoInfo (www.olst.umontreal.ca/dicoinfo). Je remercie également le groupe TecnoLeTTra de l'Université Jaume I, de Castellón dont les commentaires ont permis de clarifier certains aspects de termes d'informatique espagnols.

mc.lhomme@umontreal.ca 
Marie-Claude L'Homme est professeure au Département de linguistique et de traduction de l'Université de Montréal depuis 1994. Elle y enseigne la terminologie et les outils d'aide à la traduction. Elle est également directrice du groupe de recherche Observatoire de linguistique SensTexte (OLST). Ses travaux portent essentiellement sur la sémantique lexicale et la linguistique de corpus appliquées à la terminologie. Ils ont fait l'objet de publications dans des revues comme Terminology, Cahiers de lexicologie, International Journal of Lexicography et International Journal of Corpus Linguistics. Enfin, elle est rédactrice en chef (en collaboration avec Kyo Kageura de l'Université de Tokyo) de la revue Terminology.

\section{Bibliographie}

Bae H.-S., M.-C. L'Homme (2008, à paraître). Converting a Monolingual Database into a Multilingual Specialized Dictionary. In Proceedings of the Conference on Multilingualism and Applied Comparative Linguistics, Cambridge, Cambridge University Press.

Binon J., S. Verlinde, J. Van Dyck et A. Bertels (2000). Dictionnaire d'apprentissage du français des affaires. Dictionnaire de compréhension et de production de la langue des affaires, Paris, Didier.

Claveau V., M.-C. L'Homme (2005). Apprentissage par analogie pour la structuration de terminologie - Utilisation comparée de ressources endogènes et de ressources exogènes. In Actes. Terminologie et intelligence artificielle, TIA 2005, Université de Rouen, France.

Cohen B. (1986). Lexique de cooccurrents. Bourse-conjoncture économique. Montréal, Linguatech.

102 University Press.

Cruse D.-A. (1986). Lexical Semantics, Cambridge, Cambridge 
Drouin P. (2003). Term extraction using non-technical corpora as a point of leverage. Terminology 9(1), p. 99-117.

Fillmore C.-J. (1982). Frame Semantics. In The Linguistic Society of Korea (éd.). Linguistics in the Morning Calm, Seoul, Hanshin, p. 111137.

Fillmore C.-J., C. R. Johnson \& M.R.L. Petruck. (2003). Background to FrameNet, In Fontenelle, T. (2003). FrameNet and Frame Semantics. Special Issue of the International Journal of Lexicography 16(3), p. 235-250.

FrameNet (http://framenet.icsi.berkeley.edu/) Consulté le 14 mars 2008.

L'Homme M.-C. (1998). Définition du statut du verbe en langue de spécialité et sa description lexicographique. Cahiers de lexicologie 73(2), p. 61-84.

L'Homme M.-C. (2004). La terminologie : principes et techniques, Montréal, Les Presses de l'Université de Montréal.

L'Homme M.-C. (2008). DicoInfo. Dictionnaire fondamental de l'informatique et de l'Internet. Version Web (http://olst.ling.umontreal.caldicoinfo). Consulté le 21 mars 2008.

Mel'čuk I., A. Clas, A. et A. Polguère (1995). Introduction à la lexicologie explicative et combinatoire, Louvain-la-Neuve (Belgique), Duculot / Aupelf - UREF.

Meynard I. (2000). Internet. Répertoire bilingue de combinaisons lexicales spécialisées français-anglais, Montréal, Linguatech.

Polguère A. (2003). Collocations et fonctions lexicales : pour un modèle d'apprentissage. In Grossmann, F. et A. Tutin (dir.). Les Collocations. Analyse et traitement, coll. "Travaux et Recherches en Linguistique Appliquée », E:1, Amsterdam: De Werelt, p. 117-133.

Sager J.-C. (1990). A Practical Course in Terminology Processing, Amsterdam / Philadelphia, John Benjamins. 


\section{TRADUIRE,}

Revue trimestrielle de la Société française des traducteurs (ISSN 0395773X)

Traduire - Un regard pluriel : chaque trimestre, parole est donnée aux différents acteurs de la profession. Enquêtes, articles thématiques, entretiens et essais reflètent la diversité des points de vue des traducteurs, écrivains, donneurs d'ouvrage et théoriciens, suscitent la réflexion, élargissent le débat et enrichissent la pratique quotidienne.

Un regard actuel : tribunes libres, actes de colloques, présentation critique d'ouvrages de traductologie, dictionnaires et revues.

Traduire - Une autre perspective sur la traduction.

\section{APPEL À CONTRIBUTIONS}

\section{La Commission Traduire sollicite vos contributions !}

\section{Prochains numéros :}

- Traduction et médias

- Traduire la publicité

- Figures du traducteur

\section{D’une manière générale,} sur des sujets incluant notamment :

- Tous domaines de spécialité de la traduction

- Traduction d'édition

- Doublage, sous-titrage

- Interprétation

- Méthodologie et évaluation en traduction

- Communication interculturelle

- Comptes rendus d'ouvrages

Résumé d'article : 1000 mots

Article : 30000 à 40000 signes

Comptes rendus d'ouvrages : 5000 signes maximum (essais, dictionnaires, etc.)

Merci d'adresser vos propositions ou questions à : marie.gravey@orange.fr

ou dominique.martin.trad@wanadoo.fr 



\section{TRADUIRE \\ LBVDกIKE}

TRADUIRE publie chaque trimestre des articles d'intérêt général sur les divers aspects de la traduction, dans les domaines littéraires, scientifiques et techniques, des comptes rendus d'ouvrages, des analyses de revues spécialisées, des glossaires techniques, etc.

L'abonnement se fait par année civile avec envoi rétroactif des revues si vous vous abonnez en cours d'année. Pour l'année 2008 l'abonnement commence avec le n 216.

Pour l'étranger, les prix comprennent l'expédition par avion.

\section{TARIFS D'ABONNEMENT À LA REVUE «TRADUIRE » $\mathrm{du} \mathrm{n}^{\circ} 216$ au n $^{\circ} 219-2008$}

\begin{tabular}{|c|c|}
\hline $\begin{array}{c}\text { Adhérents SFT } \\
\text { (à jour de leur cotisation), associations } \\
\text { adhérentes à la FIT et adhérents ATLF }\end{array}$ & $40,00 €$ \\
\hline $\begin{array}{c}\text { Non-adhérents } \\
\text { résidant en France métropolitaine }\end{array}$ & $50,00 €$ \\
\hline $\begin{array}{c}\text { Non-adhérents } \\
\text { résidant hors de } \\
\text { France métropolitaine }\end{array}$ & $70,00 €$ \\
\hline
\end{tabular}

Payable en euros uniquement par :

$>$ chèque compensable sur une banque française, à l'ordre de "SFT";

> télépaiement sécurisé via la rubrique "Paiement en ligne " de notre site Internet www.sft.fr;

- virement sur le CIC - 57 rue des Martyrs - 75009 Paris.

\begin{tabular}{|c|c|c|c|c|c|}
\hline $\begin{array}{c}\text { Banque } \\
30066\end{array}$ & $\begin{array}{c}\text { Guichet } \\
10821\end{array}$ & $\begin{array}{c}\mathrm{N}^{\circ} \text { compte } \\
00010428401\end{array}$ & $\begin{array}{c}\text { Clé } \\
44\end{array}$ & FR763006 6108 2100 0104 2840 144- code BIC : CMCIFRPP \\
\hline
\end{tabular}

Pour ce dernier mode de règlement, merci de bien préciser la nature de votre paiement en indiquant en objet " Abonnement Traduire 2008 ».

Pour les personnes souhaitant se procurer d'anciens numéros de la revue, merci d'adresser votre demande par courriel à secretariat@sft.fr, afin de connaître la disponibilité des numéros souhaités, ainsi que leur prix à l'unité.

NOM

\section{BULLETIN D'ABONNEMENT À LA REVUE «TRADUIRE » 2008}

Adresse

Code Postal

Ville

Modes de règlement :

$\square$ par chèque (libellé à «SFT») $\mathrm{n}^{\circ}$

$\square$ Banque émettrice

Date d'émission

$\square$ par paiement sécurisé via la rubrique « Paiement en ligne » de notre site www.sft.fr

Date 